\title{
Morphology and Wear Resistance of Multicomponent Diffusion Coatings
}

\author{
Shun-Qi MEI ${ }^{1, a}$, Ya-Jie DONG ${ }^{1}$, A.M. Guriev, Wen-Li ZHANG ${ }^{1}$, Chao LIU ${ }^{1}$, \\ S.G. Ivanov, M.A. Guriev, T.G. Ivanova, A.M. Guriev and Chernykh E.V. \\ ${ }^{1}$ Wuhan Textile University, Wuhan, China \\ ${ }^{2}$ Altay State Technical University, Barnaul, Russia \\ ameishunqi@vip.sina.com.
}

Keywords: Wear Resistance, Microstructure, Surface Hardening, Diffusion, Steel, Boriding,

\begin{abstract}
Boronizing is one of the most effective methods of thermochemical treatment of iron-carbon alloys. Boride layers formed with the diffusion can considerably increase abrasion resistance (up to 5-30 times), heat resistance (up to 1.5-2 times) of steels. The aim of this paper is to study structure and wear resistance of diffusion layers after simultaneous saturation with boron, chromium, titanium on 1045 and D2 steels. Layer after chromium boronizing on 1045 steel has a classic needle structure of boride layer. The microstructure of layer on D2 steel is significantly different. The chromium boronizing layer has sufficiently smooth border. Globular particles of carbides and borides are relatively distributed in the iron matrix. After simultaneous saturation by boron and titanium the layer has multi-zone structure. The layer can be divided into 3 zones: upper zone consisting of titanium borides; medium zone consisting of titanium borides and carbides; bottom zone with titanium carbides. It was found that wear depends on the type of used steel. For steel D2 the diffusion layer splits off into pieces of abrasive particles, it leads to reduce durability of diffusion layer on this steel. For steel 1045 it manages to increase the resistance of parts due to the plasticity of the diffusion layers. And it is recommended that the high-alloy steel D2 can be replaced with the steel 1045 with the diffusion layer based on boron and chromium.
\end{abstract}

\section{Introduction}

Boriding diffusion is one of the most common types of chemical and heat treatment of iron-carbon alloys. Boride layers formed on the diffusion boriding steel parts considerably (5-30 fold) increase the abrasion resistance, heat resistance (1.5 - 2 fold) and corrosion resistance, which improves the life of the hardened product[1-3]. The thermal insulation coating materials were prepared by exploring the effect of amount, complex mode, and other factors of the above three kinds of functional fillers on the thermal reflection and insulation performance of coating materials [4].

Comprehensive analysis on the existing literature shows that, there are some important mecahnsim problems about diffusion coatings that have not been solved, and neede to be in-depth investigateed, such as the relationship among the life, resistance, surface hardening, morphology, and the microstructure of diffusion coatings, and this is the main topic to discuss in this paper.

\section{Materials and Methods}

We choosed steels 1045 and D2 as objects for our study. The rollers for rolling of low carbon steel were made from these steels [1-3]. Then the rollers were hardened in accordance with the recommendations for chromium boronizing and titanium boronizing [4-7]. The process was conducted and integrated by simultaneous borohromiromizing or borotitanizing isothermally at $950^{\circ} \mathrm{C}$ and held at saturation temperature for 2.5 hours in a box furnace SNOL 360/I2 12.5 .

After that the rollers were quenched (steel 1045 - water, steel D2 - oil) from the saturation temperature. followed by tempering at a low temperature of $200^{\circ} \mathrm{C}$ for 2 hours to relieve stresses in accordance with the recommendations of [6]. The boronizing powder medium contains boron in 
its composition, iron, oxygen, chromium, sodium, fluorine, chlorine, nitrogen. We used powders of boron, chromium oxygen, chlorine for the chromium boronizing process and titanium oxygen - for the titanium boronizing .

\section{Results and Discussion}

The microstructures of the layers are shown on Fig. 1. As can be seen from the figure, carbon steel microstructure boronizing layer has a classic needle appearance inherent boride coatings, while the microstructure boronizing coatings on steel D2 is significantly different in structure from the boride coatings on high-chromium steels D2 type, the boride layer on these steels is sufficiently smooth boundary.

Layer is scattered clusters of globular particles and relatively evenly distributed in the iron matrix, but virtually there is no contact between them. The upper white part of the layer is a mixture of carbides, borides and compound carbon with boron of chromium and iron, wherein the relative proportion of the iron compounds in this part of the diffusion layer is small (less than 37-40\%), while the lower part of the layer is primarily a mixture of the carbides, karboboridov and borides of iron, chromium alloy.

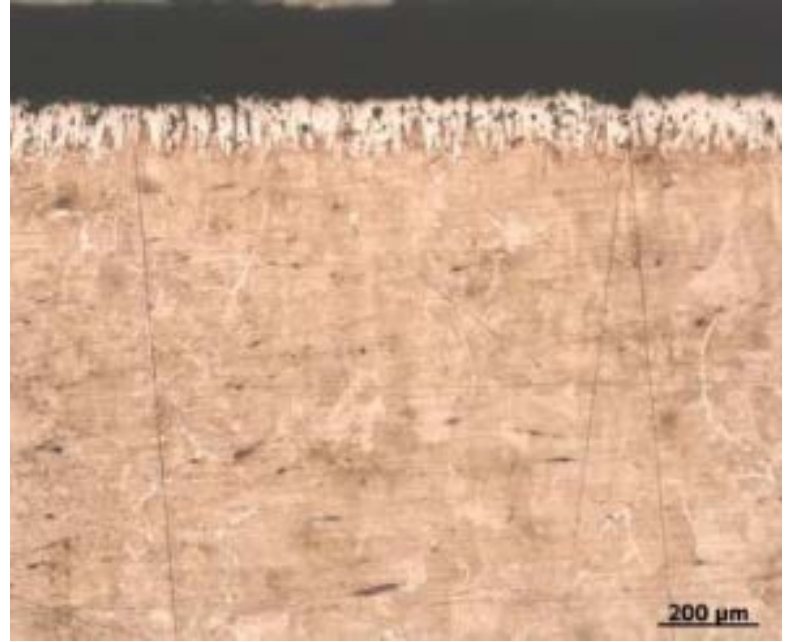

(a)

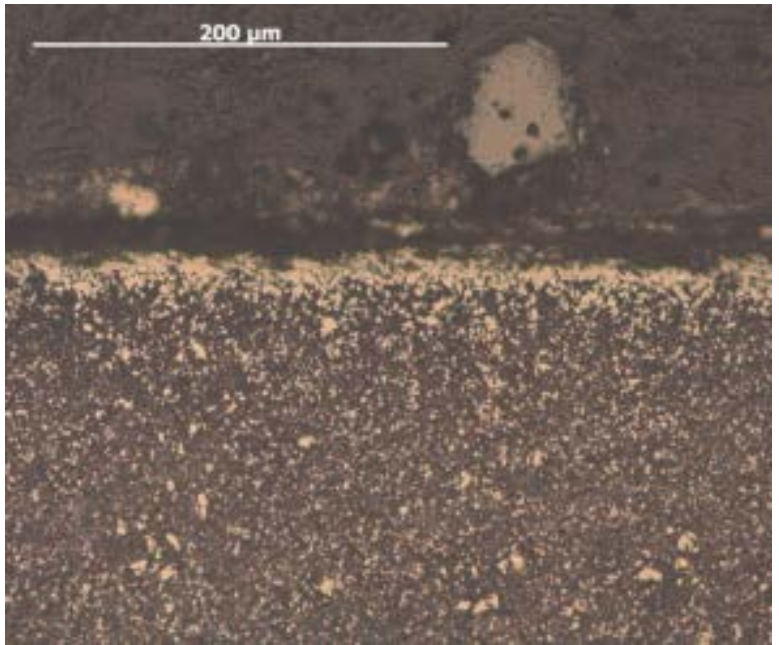

(b)

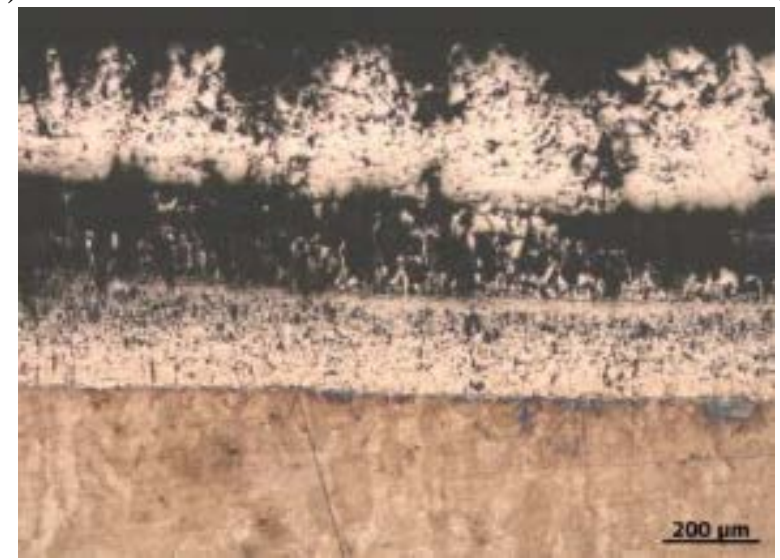

(c)

Fig. 1 Microstructures of the layers after simultaneous saturation on steels:

steel 1045 after chromium boronizing (a), steel D2 after chromium boronizing (b), steel 1045 after titanium boronizing (c)

After simultaneous saturation by boron and titanium the layer has multi-zone structure. It is shown on Fig. 1 (c). 
The layer can be divided into 3 zones: upper zone consisting of titanium borides; medium zone consisting of titanium borides and carbides; bottom zone with titanium carbides. Medium zone is fragile so it crumbles while we made the preparation of the sample. A third sublayer located under the dark layer is transient and includes titanium boride and titanium, in which the pores are titanated layer. Penultimate underlayer is titanium columnar crystals doped with boron and carbon. Directly adjacent to the carbide base material the layer consists of titanium carbide. Interfacial boundaries between phases to each other are smooth, that in combination with a high hardness and brittleness leads to poor performance of these coatings, as by applying normal and shear stresses especially cleave data layers, and their fragments, which have high hardness, are abrasive, significantly increases the wear.

Lack of titanium in the diffusion layer can be attributed to the fact that titanium, having a high affinity to non-metallic elements than iron, boron connects to the diffusion layer on the surface and forms a boride of titanium, which at the saturation temperature is not disproportionation. This supposition is confirmed by the fact that the diffraction images of the surface of the diffusion coating, obtained by X-ray diffractometer DRON 6.0 recorded only peaks of titanium, boron, carbon and their compounds, peaks of other elements and compounds not found in the diffraction patterns.

After hardening the profile roller worked in the real wear at knurled surfaces. Along with hardened knurled we also used unreinforced and rolling of steel 1045, hardened and tempered without layers. Depreciation was monitored by the change in height of the tooth, the total wear value was loss of the height more than $0.8 \mathrm{~mm}$. Thus, it should be noted also that the operation mode combines both abrasive and adhesive component wear due to friction and component wear due to the deformation of the tooth, as in the process a high contact pressure reaching $200 \mathrm{~N} / \mathrm{cm}^{2}$ is presented. It should be noted that the rolling tool refers to heavy-duty tools that work in difficult conditions. The graphic clips rolling resistance is shown in Figure 2. The profile roller was taken to decrease the height of the tooth on the value of more than $0.8 \mathrm{~mm}$, or if the tooth shape has deformation, which does not provide a clear relief knurled surface.

After testing the durability we investigated the structure of rollers, which was carried out cutting samples in the radial and axial directions. The results of metallographic studies of the worn rolling rolls, cut in the radial direction (along the length of the tooth with profile) are shown in Figure 3 and below.

As seen from the photographs of the microstructure of the material, the wear pattern depends on the type of steel which varies to a sufficiently strong extent: in the case of steel wear 1045 preferably has the form of chipping diffusion layer due to excessive loads, leading to deformation of the material of the tooth in the case of reducing the thickness of the diffusion layer, as shown there are abrupt changes in the size of the tooth at the stage of running (the time period of 0-15 minutes) and after 20 minutes of profile roller. In the case of the profile roller tool steel D2 had abrasive wear character along with the deformation of the material as a result of the high tooth loadings, the thickness of the diffusion layer in this steel grade does not exceed 60-80 microns, and moreover, the optimum temperature for the quenching of the steel is in range of $1000-1050{ }^{\circ} \mathrm{C}$, which can be coupled to form a fusible boride eutectic melting at about the same temperature range $\left(1070-1150^{\circ}\right.$ C) and a slight increase in saturation temperature above $1050^{\circ} \mathrm{C}$, the natural fluctuations of the temperature field in the volume of the furnace due to convection and non-uniformity of thermal radiation from the walls and bottom of the furnace can lead to marriage. Quenching with a selected saturation temperature of $950^{\circ} \mathrm{C}$ in steel D2 with possiblity to maintain a sufficiently high (12-15 wt.\%) of residual austenite has lowered durability characteristics, in addition, reduced temperature leads to incomplete dissolution of chromium carbides, coagulation and as consequence - impoverishment of the iron matrix alloying elements. 


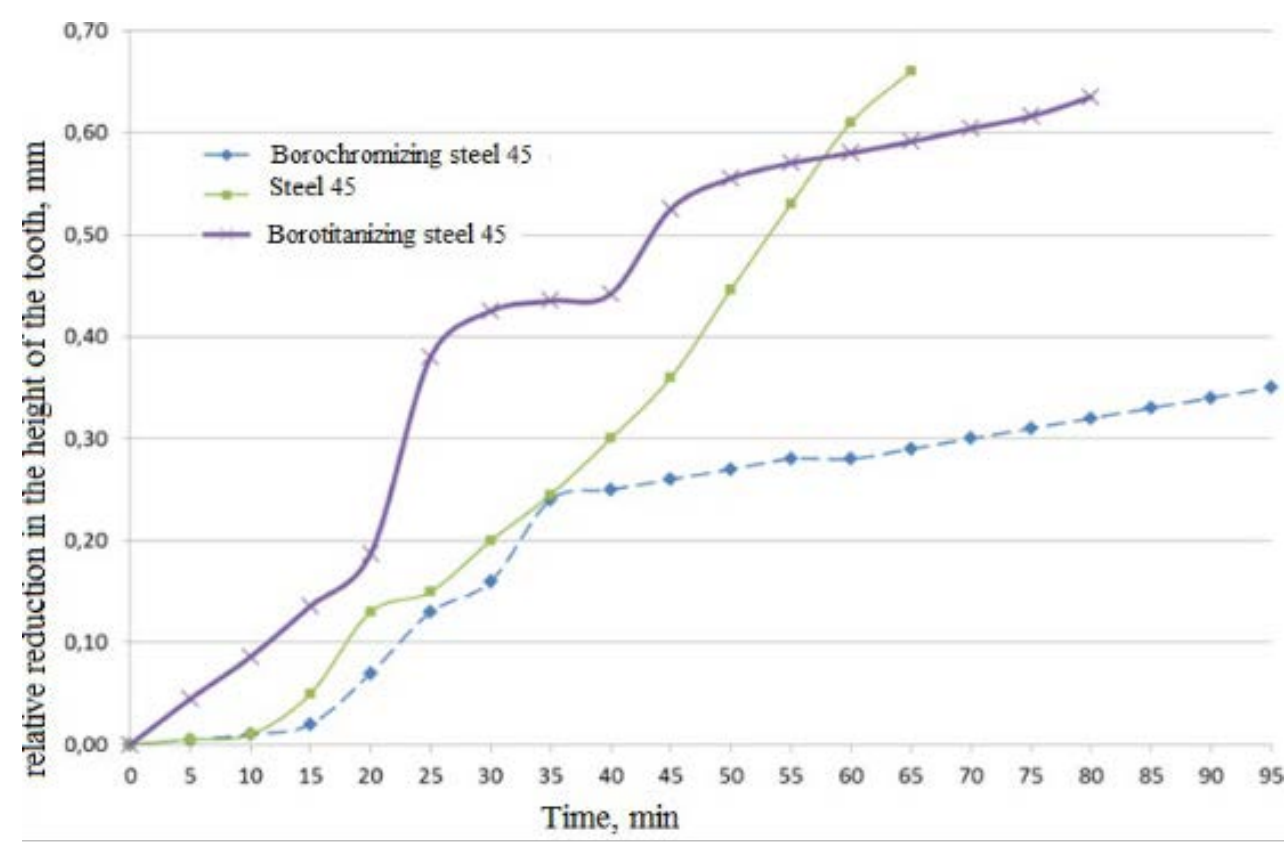

(a)

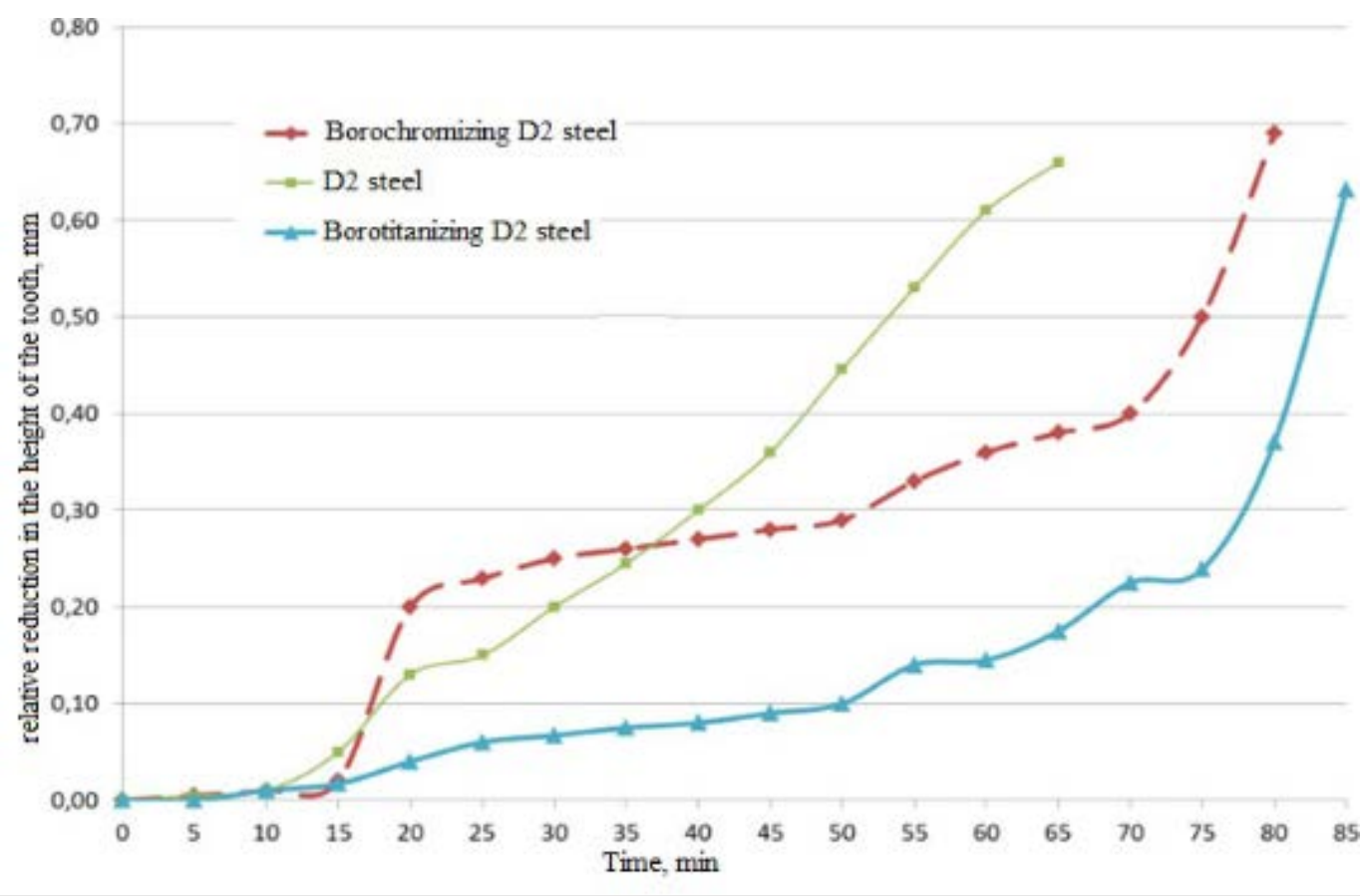

(b)

Fig. 2 The test of wear resistance of rollers: a) rollers of steel 1045, b) rollers of steel D2

The findings showed that the highest resistance profile roller carbon structural steel 45 , subjected to simultaneous diffusion boronizing, quenched with boronizing temperature and subsequent tempering with low temperature, the wear boronizing rolling rolls of steel 1045 is by 7 times higher than the durability of similar products from steel D2 , and by 8.6 times compared with wear resistance of steel rolling rolls D2, hardened and tempered with low temperature. 


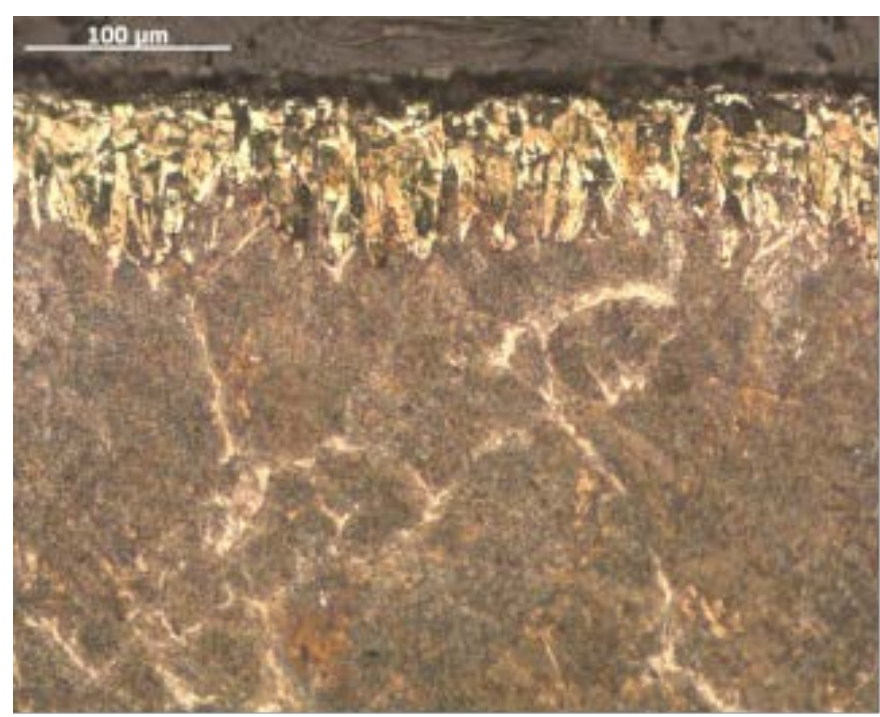

(a)

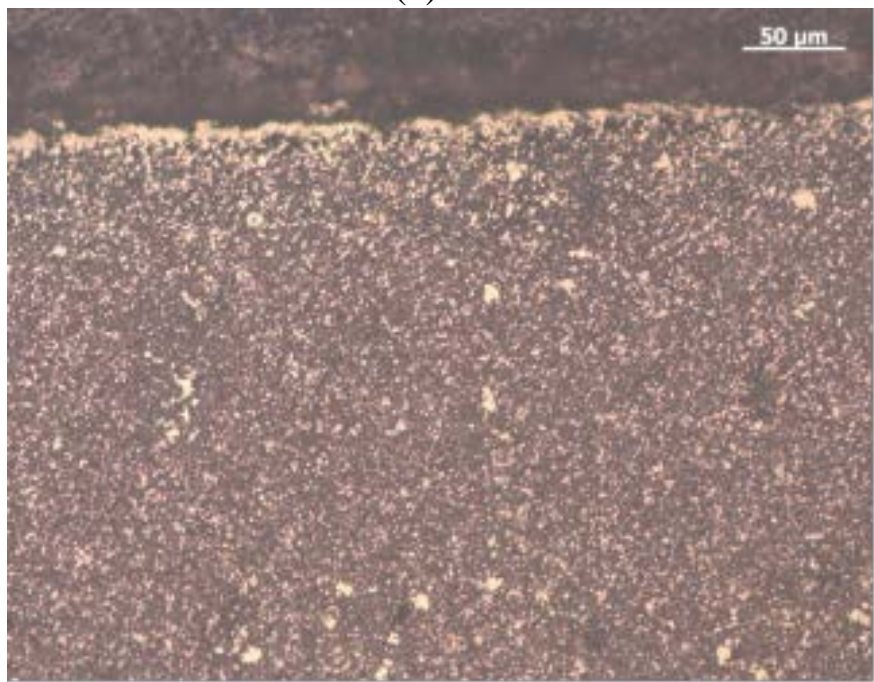

(b)

Fig. 3 Microstructures of the boride layers on a steel 1045 (a) and D2 (b) after the testing

In the manufacture of heavy duty machinery parts and tools of high-alloy steels, in order to inncrease their resistance, it can be recommended to use the thermochemical treatment (boronizing) mode with cyclically changing temperature near the temperature of phase transformation $\alpha \leftrightarrow \gamma$ that promotes intensification of diffusion processes in chemical and thermal treatment, which can lead to the dissolution of fine carbides and their uniform distribution in a matrix.

It is convinced that the best solution in this situation is the replacement of high-alloy steel D2 on carbon steel 1045 in conjunction with the diffusion boronizing. In this case, it may be received additional economic benefits due to reduced cost of production of rolling rolls and saving material and energy resources as a result of the simpler modes of mechanical, chemical-thermal and thermal processing.

\section{Conclusion}

Wear depends on the type of used steel. It was found that diffusion layer splits off into pieces when we used D2 steel. Pieces are abrasive particles. It leads to reducing durability of diffusion layer on this steel. When using steel in 1045 it is managed to increase the resistance of parts due to the plasticity of the diffusion layers. It is recommended that the high-alloy steel D2 can be replaced with the steel 1045 with the diffusion layer based on boron and chromium. 


\section{Acknowledgment}

The research work of this paper is sponsored by The Science \& Technology Research Plan of Hubei province government with project [2012AAA07-02] and [2014BHE010].

\section{References}

[1] Guriev A.M. Physical basis of chemical and thermal cycling treatment of steel, Lygdenov B.D., Popova N.A., Kozlov E.V. Barnaul, 2008 (in Russian).

[2] Guriev A.M. Structure and properties of hardened boron and boron together with the titanium surface and die steels 5HNV 5H2NMVF. Ivanov S.G., Guriev M.A., Ivanov A.G., Lygdenov B.D., Zemlyakov S.A., Dolgorov A.A. Fundamental problems of modern materials. 7(1): 27-31 (in Russian).

[3] Guriev A.M. The mechanism of diffusion of boron, chromium and titanium, while the multi-component saturation of the surface iron-carbon alloys. Ivanov S.G. Fundamental problems of modern materials. 8(3): 92-96 (in Russian).

[4] Wang F, Liang J, Tang Q, Chen G, Chen Y. Preparation and performance of thermal insulation energy saving coating materials for exterior wall. J Nanosci Nanotechnol. 2014 May; 14(5):3861-7.

[5] Guriev A.M. Diffusion thermocyclic surface hardening of steel products with boron, titanium and chromium. Lygdenov B.D., Ivanov S.G., Vlasova O.A., Kosheleva E.A., Garmaeva I.A., Guriev M.A. Fundamental problems of modern materials. 4(1): 30-35 (in Russian).

[6] Ivanov SG, Guriev AM, Starostenkov MD, Ivanova TG, Levchenko AA Special features of preparation of saturating mixtures for diffusion chromoborating. Russian Phys J 57(2): 266-269. DOI: $10.1007 / \mathrm{s} 11182-014-0234-6$.

[7] Guriev A.M. The multicomponent diffusion hardening surfaces of machine parts and tools from mixtures based on boron carbide. Greshilov A.D., Kosheleva E.A., Ivanov S.G., Guriev M.A., Ivanov A.G. ., Dolgorov A.A. Metal Finishing: technology, equipment, tools. 2010(2): 19-23.

[8] Ivanov S.G. Features of Multicomponent Saturation Alloyed by Steels. I.A. Garmaeva, M.A. Guriev, A.M. Guriev, M.D. Starostenkov \& A.N. Evgrafov (eds). Advances in Mechanical Engineering. Lecture Notes in Mechanical Engineering. 2015: 49-53. DOI: 10.1007/978-3-319-15684-2_7

[9] Guriev A.M. The mechanism of formation of boride needles with someone diffusion of saturating the complex borohromirovanii plasters. Ivanov S.G., Greshilov A.D., Zemlyakov S.A. Metal Finishing: technology, equipment, tools. 2011(3): 34-40.

[10] Guriev M.A. The technology of multi-hardening coatings on steel parts. Filchakov D.S., Garmaeva I.A., Ivanov S.G., Sasha A.M., Okolovich G.A. Polzunovsky Gazette. 2012.1(1): 73-78.

[11] Guriev A.M. Diffusion coating of steels and alloys. Ivanov S.G., Garmaeva I.A. Barnaul, 2013. 